Noname manuscript No.

(will be inserted by the editor)

\title{
Takuma Akimoto \\ Generalized Arcsine Law and Stable Law in an Infinite Measure Dynamical System
}

Received: date / Accepted: date

\begin{abstract}
Limit theorems for the time average of some observation functions in an infinite measure dynamical system are studied. It is known that intermittent phenomena, such as the Rayleigh-Benard convection and Belousov-Zhabotinsky reaction, are described by infinite measure dynamical systems. We show that the time av, erage of the observation function which is not the $L^{1}(m)$ function, whose average with respect to the invariant measure $m$ is finite, converges to the generalized arcsine distribution. This result leads to the novel view that the correlation function is intrinsically random and does not decay. Moreover, it is also numerically shown that the time average of the observation function converges , to the stable distribution when the observation function has the infinite mean.
\end{abstract}

Keywords Non-stationary Chaos - Infinite measure · Generalized Arcsine Law · Non-equilibrium state

\section{Introduction}

Recently, the $1 / f$ power spectrum and the power law phenomena, which are closely related to the intermittent phenomena [1, have been studied in various systems. Examples are the $1 / f$ power spectrum in the RayleighBenard convection [2], Belousov-Zhabotinsky reaction [3, fluorescence intermittency in single nanocrystals 4 and the power law decay of the earthquake phenomena [5, 6. It is also known that such power law phenomena are clearly observed in Hamiltonian systems and nonhyperbolic dynamical systems 7,8 . It is a remarkable problem that the theoretical meaning of the $1 / f$ power spectrum has not been completely elucidated.

\section{T. Akimoto}

Department of Applied Physics, Advanced School of Science and Engineering, Waseda University, Okubo 3-4-1, Shinjukuku, Tokyo 169-8555, Japan.

Tel.: +81-3-3200-2457

Fax: +81-3-3200-2457

E-mail: akimoto@aoni.waseda.jp
In ergodic theory the time average can be replaced by the ensemble average. However, in the intermittent phenomena, this replacement is not always guaranteed. Actually, it has been pointed out that the time average of some observation shows anomalous behaviour, that is, the time average does not converge to a constant value and becomes intrinsically random [9, 10. Therefore it is important to analyze the behaviour of the time average in the intermittent phenomena from the viewpoint of ergodic theory. Infinite measure dynamical systems are examples of dynamical systems describing such intermittent phenomena. The recent study of infinite ergodic theory tells us that the time average of some observation functions converges in distribution [11,12,13, 14. For example, the scaled time average of the $L^{1}(m)$ function $g(x), \sum_{k=0}^{n-1} g\left(T^{k} \cdot\right) / a_{n}$, converges to the MittagLeffler distribution, where $a_{n}$ is the proper sequence.

The purpose of this paper is to study the time average of the non- $L^{1}(m)$ function in infinite measure systems. In the previous works, it is already known that the distribution of the occupation time of some interval in infinite measure systems converges to the generalized arcsine distribution [15, 16. In this paper we clear the class of the observation function whose time average converges to the generalized arcsine distribution using the modified Bernoulli map which is the typical example of a one-dimensional infinite measure dynamical system on $[0,1]$.

The paper is organized as follows. In $\S 2$ we review the modified Bernoulli map. In $\S 3$ we show the distribution for the time average of the $L_{l o c}^{1}(0,1)$ function with finite mean using a modified Bernoulli map. Theorem 1 and Theorem 2 are the generalized arcsine laws, which are well known in random process, in the dynamical system. Theorem 3, which is the main result in this paper, generalizes the observation function. In $\S 4$ our results are applied to the correlation functions. In $\S 5$ we numerically demonstrate the distribution of for the time average of the $L_{l o c}^{1}(0,1)$ function with infinite mean. $\S 6$ is devoted 
to summary and an approach toward the ergodic problems of non-equilibrium statistical mechanics.

\section{Reviews of the modified Bernoulli map}

In this section we review the statistical properties of the modified Bernoulli map. The modified Bernoulli map is one dimensional map on $[0,1]$ defined by

$$
\begin{aligned}
x_{n+1} & =T x_{n} \\
& = \begin{cases}x_{n}+2^{B-1} x_{n}^{B} & x_{n} \in I_{0}=[0,1 / 2] \\
x_{n}-2^{B-1}\left(1-x_{n}\right)^{B} & x_{n} \in I_{1}=(1 / 2,1] .\end{cases}
\end{aligned}
$$

This map has two indifferent fixed points $(x=0$ and 1$)$, at which the invariant density $\rho(x)$ is not bounded. The invariant density $\rho(x)$ can be written as

$\rho(x) \sim x^{1-B}+(1-x)^{1-B}$

for $x \sim 0$ and $x \sim 1$. Therefore for $B \geq 2$ the invariant density cannot be normalized, that is, the invariant measure becomes the infinite one [7, 17, 18.

It is important that renewal processes are constructed by the sequences of the modified Bernoulli map [19. Actually, using the symbolic sequence $\sigma_{n}=\sigma\left(x_{n}\right)$, where $\sigma(x)=-1\left(x \in I_{0}\right)$ and $\sigma(x)=1\left(x \in I_{1}\right)$, one can define the renewal when the value of $\sigma_{n}$ changes, namely $\sigma_{n} \sigma_{n+1}=-1$. As the time interval between $r-1$ th and $r$ th renewal denoted by $\mathbf{X}_{r}(r \geq 2)$ is an independently identically distributed random variable, and this probability density function (p.d.f.) $f(n)$ is given by $f(n) \propto(n-1)^{-\beta}(\beta=B /(B-1))$ for $n \gg 1$ [20], this process is regarded as a renewal process. In what follows, we consider the time interval between successive renewals as continuous random variables, i.e., p.d.f. is given by

$$
f(x)=(\beta-1)(x-1)^{-\beta} \quad(\beta=B /(B-1)) .
$$

It is noted that the p.d.f. of the first renewal time $\mathbf{X}_{1}$ depends on the initial ensemble of the modified Bernoulli map 21,22. When the initial ensemble is the invariant density for the first passage map $T^{n(x)}(x)$ with respect to $E=\left[e_{1}, e_{2}\right]$, whose endpoints are the solutions of the equation $T x=1 / 2$ for $e_{1}<e_{2}$ and $n(x)=1+\min \{n \geq$ $\left.0: T^{n}(x) \in E\right\}$, the p.d.f. of $\mathbf{X}_{1}$ is same as Eq. (3), namely the ordinary renewal process. However, when the initial ensemble is the invariant density for the modified Bernoulli map, the p.d.f. of the first renewal time $\mathbf{X}_{1}$ is given by $f_{1}(x)=(1-F(x)) / \mu$, which is completely different from Eq. (3), where $F(x)$ is the cumulative distribution function of $f(x)$ and $\mu$ is the mean value of $\mathbf{X}_{r}$ [19]. In the previous papers 21,22] we clearly demonstrate the dependence of the statistical laws, namely, the renewal function and the correlation function, on the initial ensemble.

\section{Generalized arcsine law}

Firstly, we analyze the behaviour of the time average of the following function:

$I(x)=\left\{\begin{array}{l}a\left(x \leq \frac{1}{2}\right) \\ b\left(x>\frac{1}{2}\right),\end{array}\right.$

where $a, b \in \mathbb{R} \backslash\{-\infty, \infty\} \mathbb{1}$ It is noted that $I(x)$ is not the $L^{1}(m)$ function.

We review Lamperti's generalized arcsine law for the modified Bernoulli map [24].

Theorem 1 (Lamperti's generalized arcsine law) Let $\mathbf{X}_{n}$ be the time interval between the successive renewals in the renewal process constructed by the modified Bernoulli map and $N_{n}$ be the occupation time in $I_{0}$, that is, $N_{n}=\mathbf{X}_{1}+\mathbf{X}_{3}+\ldots+\mathbf{X}_{n}$ when $x_{0} \in I_{0}$ and $n$ is odd. Then

$\lim _{n \rightarrow \infty} \operatorname{Pr}\left(N_{n} / n \leq x\right)=G_{\alpha}(x)$

exists, where $\alpha=\beta-1$ and the p.d.f. $G_{\alpha}^{\prime}(x)$ is given by

$G_{\alpha}^{\prime}(x)=\frac{\sin \pi \alpha}{\pi} \frac{x^{\alpha}(1-x)^{\alpha-1}+x^{\alpha-1}(1-x)^{\alpha}}{x^{2 \alpha}+2 x^{\alpha}(1-x)^{\alpha} \cos \pi \alpha+(1-x)^{2 \alpha}} \cdot(6)$

The distribution $G_{\alpha}(x)$ is called the generalized arcsine distribution.

Proof. In 24] the limit probability (5) exists if and only if there exist constants $c$ and $\alpha$ such that

$\lim _{n \rightarrow \infty} E\left(N_{n} / n\right)=c$

and

$\lim _{x \rightarrow 1-} \frac{(1-x) u^{\prime}(x)}{1-u(x)}=\alpha$,

where $E($.$) is the ensemble average with respect to the$ initial density and $u(x)=\sum_{n=1}^{\infty} f(n) x^{n}$ is the generating function of the $f(n)$. In what follows, we check the above conditions.

First, we define the average of the function $1_{[0,1 / 2)}\left(T^{n} x\right)$ as

$E_{n} \equiv E\left(1_{[0,1 / 2)}\left(T^{n} x\right)\right)$

According to 25 the initial density $\rho_{0}(x)$ converges to the invariant density under proper normalization:

$w_{n} P^{n} \rho_{0}(x) \rightarrow\left(\frac{1}{\Gamma(\alpha) \Gamma(2-\alpha)}\right) \rho(x)$ as $n \rightarrow \infty$,

where $P$ is the Perron-Frobenius operator and

$w_{n} \sim \begin{cases}\log n & (B=2) \\ n^{1-\alpha} & (B>2),\end{cases}$

where $\alpha=\beta-1$. The invariant density $\rho(x)$ is symmetric with respect to the axis $x=1 / 2$. Therefore

$E_{n} \rightarrow \frac{1}{2}$ as $n \rightarrow \infty$

1 The special case, namely $a=-1$ and $b=1$, is shown by using the renewal theory in 23 . 
and

$E\left(N_{n} / n\right)=\frac{1}{n} \sum_{k=1}^{n} E_{k} \rightarrow \frac{1}{2}$ as $n \rightarrow \infty$.

By Karamata's Tauberian theorem it is easily confirmed that the condition (8) holds when $\alpha=\beta-1$ :

$\lim _{x \rightarrow 1-} \frac{(1-x) F^{\prime}(x)}{1-F(x)}=\beta-1$.

The p.d.f. of $N_{n} / n$ in [24] under $c=1 / 2$ and $\alpha=\beta-1$ implies the p.d.f. (6).

Using Theorem 1 one can know the distribution of the time average of $I(x)$ immediately.

Theorem 2 The time average of $I(x)$ converges in distribution:

$\frac{1}{n} \sum_{k=0}^{n-1} I\left(T^{k} \cdot\right) \rightarrow Y_{\alpha, a, b}$

where the random variable $Y_{\alpha, a, b}$ has the following p.d.f,

$G_{\alpha, a, b}^{\prime}(x)=$

$\begin{cases}\frac{(a-b) \sin \pi \alpha}{\pi} \frac{(x-b)^{\alpha-1}(a-x)^{\alpha-1}}{(x-b)^{2 \alpha}+2(x-b)^{\alpha}(a-x)^{\alpha} \cos \pi \alpha+(a-x)^{2 \alpha}} & (a>b) \\ \frac{(b-a) \sin \pi \alpha}{\pi} \frac{(b-x)^{\alpha-1}(x-a)^{\alpha-1}}{(b-x)^{2 \alpha}+2(b-x)^{\alpha}(x-a)^{\alpha} \cos \pi \alpha+(x-a)^{2 \alpha}} & (a<b),\end{cases}$

where $\alpha=\beta-1$, that is, the random variables $Y_{\alpha, a, b}$ obeys the generalized arcsine law.

Proof. The time average of $I(x)$ can be rewritten as

$\frac{1}{n} \sum_{k=0}^{n-1} I\left(T^{k} x\right)=\frac{a N_{n}+b\left(n-N_{n}\right)}{n}$.

Using Theorem 1, we can write

$$
\begin{array}{r}
\operatorname{Pr}\left\{\frac{1}{n} \sum_{k=0}^{n-1} I\left(T^{k} x\right) \leq x\right\}=\operatorname{Pr}\left\{(a-b) \frac{N_{n}}{n}+b \leq x\right\} \\
\rightarrow\left\{\begin{array}{ll}
G_{\alpha}\left(\frac{x-b}{a-b}\right) & (a>b) \\
1-G_{\alpha}\left(\frac{x-b}{a-b}\right) & (a<b) .
\end{array} \text { as } n \rightarrow \infty\right.
\end{array}
$$

The derivative of (18), which is the p.d.f. of the time average of $I(x)$ denoted by $G_{\alpha, a, b}$, gives (16).

Definition 1 ( $L_{l o c, m}^{1}$ function with finite mean) If the following conditions

$\lim _{\epsilon \rightarrow 0} \frac{\int_{\epsilon}^{1-\epsilon}|g| d m}{\int_{\epsilon}^{1-\epsilon} d m}<\infty$

and for all $\epsilon>0$

$\int_{\epsilon}^{1-\epsilon}|g| d m<\infty$

hold, then the function $g$ is called the $L_{l o c}^{1}(0,1)$ function with finite mean with respect to $m$, denoted by $L_{l o c, m}^{1}(0,1)$ function with finite mean.

Examples of the $L_{l o c}^{1}(0,1)$ function w.r.t. the invariant measure of the modified Bernoulli map are $I(x)$ and $g(x)=x 2^{2}$

2 Roughly speaking, we can say the $L_{l o c, m}^{1}(0,1)$ function with finite mean is considered as the $L^{\infty}(0,1)$ function.
Remark 1 In the case of the modified Bernoulli map the measure of the sets $[0, \epsilon]$ and $[1-\epsilon, 1]$ are not finite. Therefore we exclude these sets in Eqs. (19) and (20). When the measure of the other sets are not finite, Equations (19) and (20) must be changed to exclude these sets.

Theorem 3 Let $g(x)$ be the $L_{\text {loc }}^{1}(0,1)$ function with $f$ nite mean w.r.t. the invariant measure $m$ and $g(0)=$ $a, g(1)=b$. Further, there exists $\delta$ such that $0<\delta<1$ and $g(x)$ is continuous in $[0, \delta] \cup[1-\delta, 1]$. Then the time average of $g(x)$ converges in distribution to $Y_{\alpha, a, b}$, where $\alpha=\beta-13$

Proof. It is shown that the time average of the $L_{+}^{1}(m)$ function 4 converges to zero [12], that is, for all $f \in$ $L_{+}^{1}(m)$

$\frac{1}{n} \sum_{k=0}^{n-1} f\left(T^{k} x\right) \rightarrow 0 \quad$ as $n \rightarrow \infty$ for almost all $x$.

By the theorem (21), for all $\epsilon>0$ there exist $N_{1}$ such

6) that for $n>N_{1}$

$$
\begin{aligned}
& \left|\frac{1}{n} \sum_{k=0}^{n-1} g_{\delta, a, b}\left(T^{k} x\right)-\frac{1}{n} \sum_{k=0}^{n-1} I\left(T^{k} x\right)\right| \\
& <\frac{1}{n} \sum_{k=0}^{n-1}\left|g_{\delta, a, b}\left(T^{k} x\right)-I\left(T^{k} x\right)\right| \\
& <\epsilon / 2
\end{aligned}
$$

where

$g_{\delta, a, b}(x)= \begin{cases}a & \text { for } x \in[0, \delta) \\ g(x) & \text { for } x \in[\delta, 1-\delta] \\ b & \text { for } x \in(1-\delta, 1]\end{cases}$

Then by the continuity of $g(x)$ and (21), for all $\epsilon>0$ there exist $\delta_{*}$ and $N_{2}$ such that for $n>N_{2}$

$\left|\frac{1}{n} \sum_{k=0}^{n-1} g\left(T^{k} x\right)-\frac{1}{n} \sum_{k=0}^{n-1} g_{\delta_{*}, a, b}\left(T^{k} x\right)\right|<\epsilon / 2$.

Therefore for all $\epsilon>0$ there exists $\delta_{*}$ and $N$ such that for $n>N$

$\left|\frac{1}{n} \sum_{k=0}^{n-1} g\left(T^{k} x\right)-\frac{1}{n} \sum_{k=0}^{n-1} I\left(T^{k} x\right)\right|<\epsilon$.

By Theorem 2, the time average of $g(x)$ converges in distribution to $Y_{\alpha, a, b}$.

Figures 1, 2] and 3 demonstrate numerically that the p.d.f. for the time average of $g(x)=x^{2}$ obeys the theoretical one even when the time $n$ is finite $\left(n=10^{8}\right)$.

3 The following proof is not changed even if the condition of $g(x)$ changes from the $L_{l o c, m}^{1}(0,1)$ function to the $L^{\infty}(0,1)$ function.

4 The $L_{+}^{1}(m)$ function is the $L^{1}(m)$ function whose value is positive. 


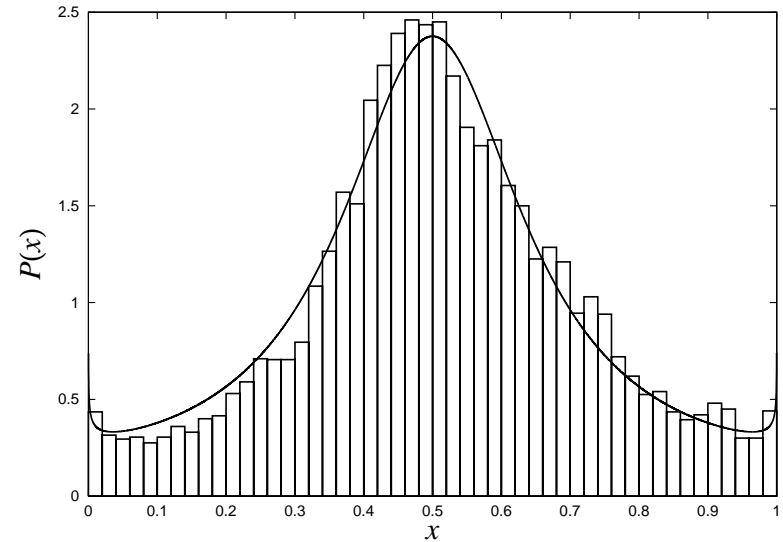

Fig. 1 The probability density function for the time average of $g(x)=x^{2}(B=2.2)$.

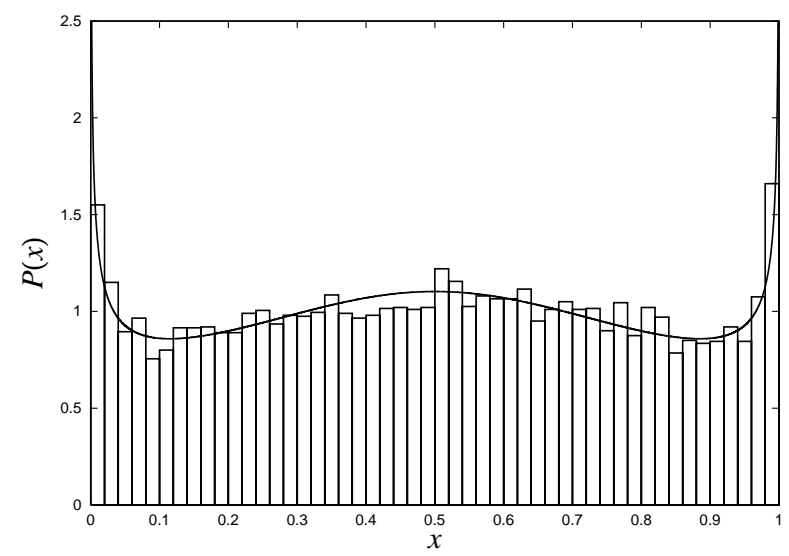

Fig. 2 The probability density function for the time average of $g(x)=x^{2}(B=2.5)$.

\section{Application to the correlation functions}

We apply Theorem 3 to the correlation function, which is defined by the time average:

$C(n)=\lim _{N \rightarrow \infty} \frac{1}{N} \sum_{k=0}^{N-1} g(x) h\left(T^{n} x\right)$.

In the case of finite measure, ergodic theory states that the correlation function defined by the time average equals to the correlation function defined by the ensemble average, namely the average of $g(x) h\left(T^{n} x\right)$ with respect to the invariant measure. However, when the invariant measure is not finite, the time-averaged correlation function is not equal to the ensemble-averaged correlation function 5 In this section we demonstrate that the correlation function is intrinsically random in the modified Bernoulli map.

\footnotetext{
5 The dependence of the decay of the ensemble-averaged correlation function on the initial ensemble is discussed in 22 .
}

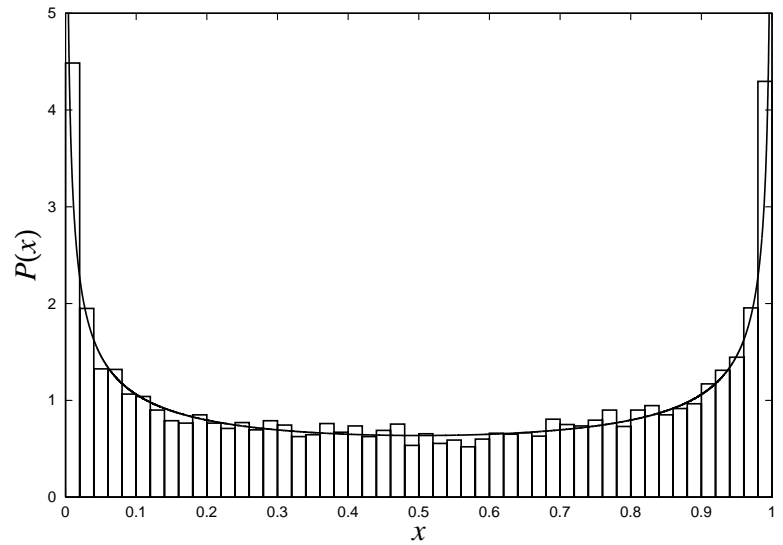

Fig. 3 The probability density function for the time average of $g(x)=x^{2}(B=3.0)$.

Corollary 1 For all $n$ the correlation function of $\sigma(x)$ converges to 1 :

$C(n)=\lim _{N \rightarrow \infty} \frac{1}{N} \sum_{k=0}^{N-1} \sigma\left(x_{k}\right) \sigma\left(x_{k+n}\right)=1$,

where $\sigma(x)=1(x \in[0,1 / 2)),-1(x \in[1 / 2,1])$.

Proof. For all $n$ the observation function $g_{n}(x)$ is defined as

$$
\begin{aligned}
g_{n}(x) & =\sigma(x) \sigma\left(T^{n} x\right) \\
& = \begin{cases}+1 & x \in\left[0, a_{n}\right] \cup\left[1-a_{n}, 1\right] \cup A_{n}, \\
-1 & \text { otherwise }\end{cases}
\end{aligned}
$$

where $a_{n}=a_{n+1}+2^{B-1} a_{n+1}^{B}\left(a_{n}>0\right.$ and $\left.a_{0}=1 / 2\right)$ and $A_{n}$ is the set which attains $\sigma(x) \sigma\left(T^{n} x\right)=1$ and is subset of $\left[a_{n}, 1-a_{n}\right]$ :

$A_{n}=\left\{x \in\left[a_{n}, 1-a_{n}\right]: \sigma(x) \sigma\left(T^{n} x\right)=1\right\}$.

Then $g_{n}(0)=g_{n}(1)=1$ and $g_{n}(x)$ is continuous in $\left[0, a_{n}\right] \cup\left[1-a_{n}, 1\right]$ and for all $\epsilon>0$

$\int_{\epsilon}^{1-\epsilon} g_{n}(x) d m<\int_{\epsilon}^{1-\epsilon} d m<\infty$.

By Theorem 3, $C(n)$ is convergence in distribution to $Y_{\alpha, 1,1}$ for all $n$.

Corollary 2 For all $n$ the correlation function of $x$ is convergence in distribution to $Y_{\alpha, 0,1}$ :

$C(n)=\lim _{N \rightarrow \infty} \frac{1}{N} \sum_{k=0}^{N-1} x_{k} x_{k+n} \rightarrow Y_{\alpha, 0,1}$

Proof. For all $n$ the observation function $g_{n}(x)$ is defined as

$g_{n}(x)=x\left(T^{n} x\right)$,

and for all $\epsilon>0$

$\int_{\epsilon}^{1-\epsilon} g_{n}(x) d m<\int_{\epsilon}^{1-\epsilon} d m<\infty$. 
Then $g_{n}(0)=0$ and $g_{n}(1)=1$, and $g_{n}(x)$ is continuous in $\left[0, a_{n}\right] \cup\left[1-a_{n}, 1\right]$. By Theorem $3, C(n)$ is convergence in distribution to $Y_{\alpha, 0,1}$ for all $n$.

Figures 4 and 5 show that the correlation functions with fixed time difference $n$ are intrinsically random and these distributions obey the generalized arcsine distribution.

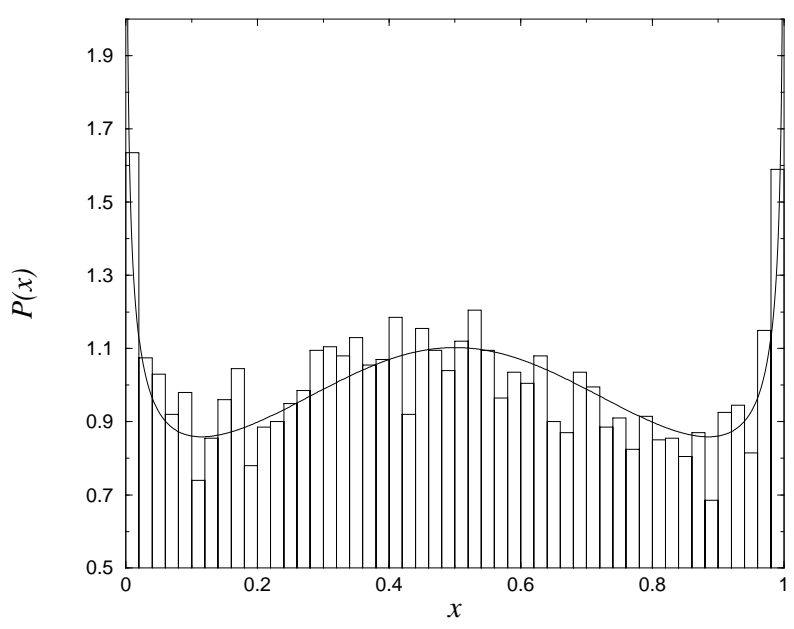

Fig. 4 The probability density function for the correlation function defined by the time average of $g_{10}(x)=x x_{10}(B=$ $2.5)$.

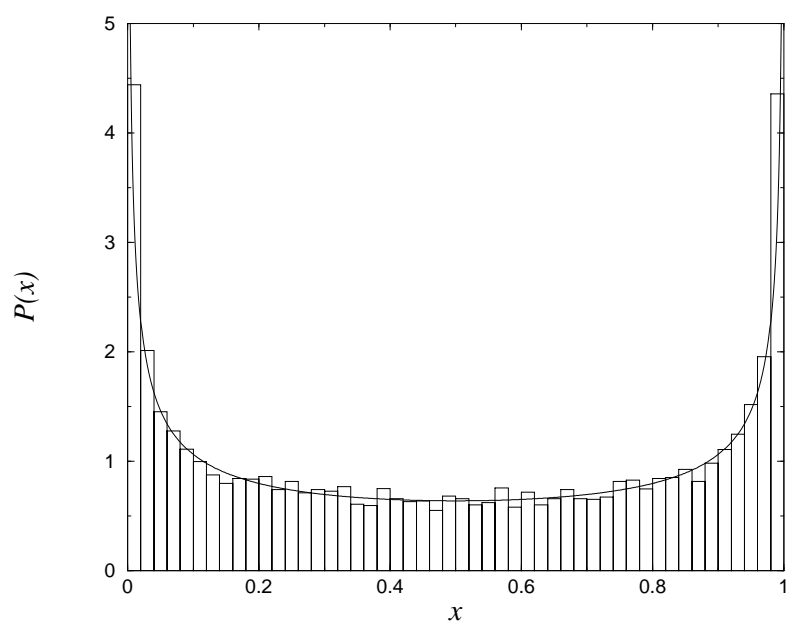

Fig. 5 The probability density function for the correlation function defined by the time average of $g_{10}(x)=x x_{10}(B=$ $3.0)$.

\section{Stable law}

In this section we demonstrate the distribution for the time average of the $L_{l o c}^{1}(0,1)$ function with infinite mean, which satisfies the condition (20) and

$\lim _{\epsilon \rightarrow 0} \frac{\int_{\epsilon}^{1-\epsilon}|g| d m}{\int_{\epsilon}^{1-\epsilon} d m}=\infty$

In the case of the finite measure, the time average of the $L_{\text {loc }}^{1}(0,1)$ function with infinite mean converges to the stable distribution, as shown in Appendix A.

In what follows, we study numerically the distribution for the time average of the observation function

$g(x)= \begin{cases}x^{-\alpha} & \left(x<\frac{1}{2}\right) \\ (1-x)^{-\alpha} & \left(x \geq \frac{1}{2}\right),\end{cases}$

which is the $L_{l o c}^{1}(0,1)$ function with infinite mean and $\alpha>0$. As shown in Fig. 6, we find that the distribution for the scaled time average of $L_{l o c}^{1}(0,1)$ function with infinite mean also converges to the stable distribution with index $\gamma$. In numerical simulations we calculate the time average of $g(x)$ for three different length of the simulation time $\left(n=10^{4}, 10^{5}\right.$ and $\left.10^{6}\right)$ and then determine the exponent $\gamma$ so as to make the distributions of the scaled time average invariant. Figure 7 shows the linear relation between $\gamma$ and $\alpha$ clearly. Moreover, we find that the scaling exponent $\gamma$ obeys the non-trivial relation to the exponent $\alpha$ and $B$ except for the case $B<2.5$, i.e.,

$\gamma=\frac{\alpha}{B-1}+1$.

This relation is clearly shown in Fig. 8 except for the case $B<2.5$. 6 We summarize these results as following conjecture.

Conjecture 1 Let $g(x)$ be the $L_{\text {loc }}^{1}(0,1)$ function with infinite mean w.r.t. the invariant measure $m$. Further,

$x^{\alpha} g(x)=O(1), \quad x \rightarrow 0$

$(1-x)^{\alpha} g(x)=O(1), \quad x \rightarrow 1$

Then the scaled time average of $g(x)$ converges to the stable distribution $G_{\gamma}$ :

$\frac{1}{b_{n}} \sum_{k=0}^{n-1} g\left(T^{k} \cdot\right) \rightarrow G_{\gamma}$

where $b_{n} \propto n^{\gamma}$ and $\gamma=\frac{\alpha}{B-1}+1$.

Remark 2 In the case of $B=2.0$ the scaling sequence $b_{n}$ had better be $n^{\gamma} / \log n$ rather than $n^{\gamma}$.

Remark 3 The distribution for the scaled time average of $g(x)=x^{-\alpha}$ also converges to the stable distribution when the invariant measure is finite. On the other hand, the distribution for the scaled time average of $g(x)$ is different from the stable one due to the generalized arcsine law for the occupation time of the interval $[1 / 2,1]$.

\footnotetext{
6 The reason that the relationship (36) does not hold for the case $B<2.5$ seems to be that the observation time is enoug in numerical simulations.
} 


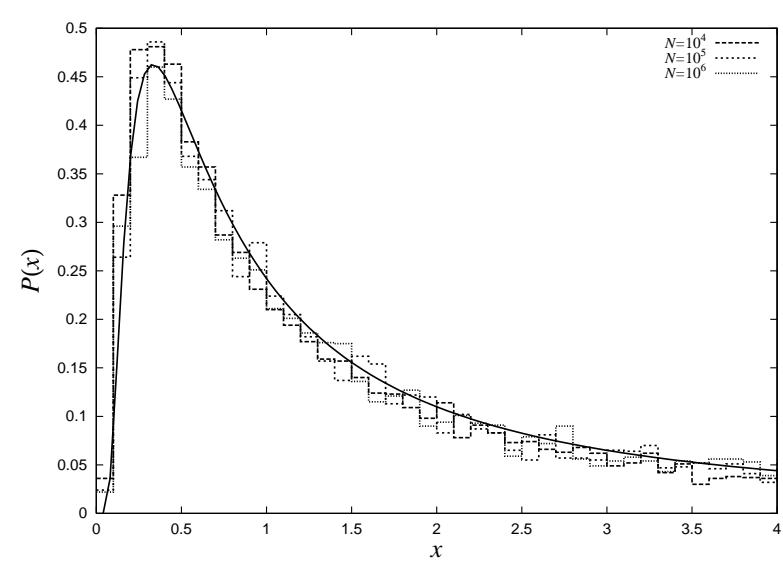

Fig. 6 The probability density function for the scaled time average of $g(x)(B=3.0$ and $\alpha=2.0)$. The fitting curve is a stable distribution with $\gamma=2.0\left(P(x)=\frac{1}{\sqrt{2 \pi x^{3}}} e^{-1 /(2 x)}\right)$.

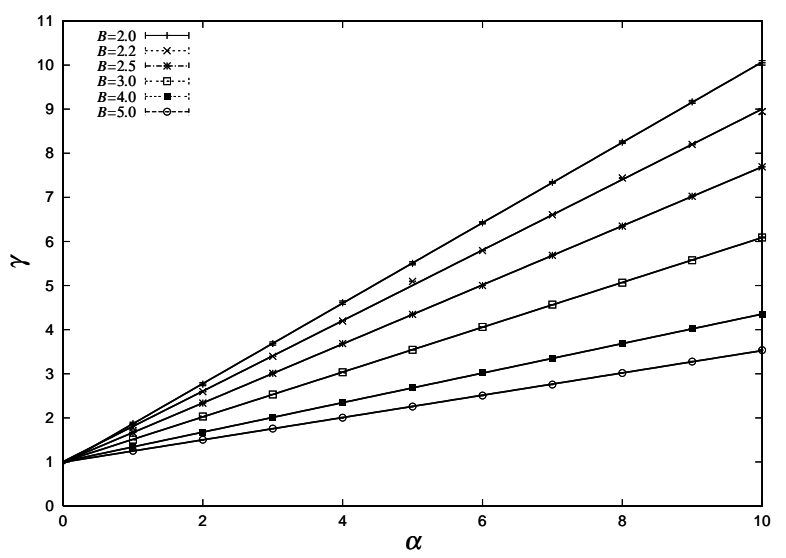

Fig. 7 Scaling exponent $\gamma$ vs. $\alpha$.

\section{Discussion}

In this paper we present the distributional limit theorems for the time average of the $L_{l o c, m}^{1}(0,1)$ function with finite mean and infinite mean using the modified Bernoulli map whose invariant measure is infinite. By applying this theorem to the correlation function, it is clearly shown that the correlation functions with fixed time difference $n$ converge to the generalized arcsine distribution. G. Margolin and E. Barkai analyzed the distribution for the correlation function of a dichotomous random process and its convergence process [10. Our results correspond to the generalized result of their work in the way that the observation function can be extended to the $L_{l o c, m}^{1}(0,1)$ function with finite mean 7 However, the convergence process of the time average of the $L_{l o c, m}^{1}(0,1)$ with finite mean is not studied. So we will study the convergence

7 In [10] the observation function is the characteristic function $I(x)$ with $a=1$ and $b=0$, which is the special case of our results.

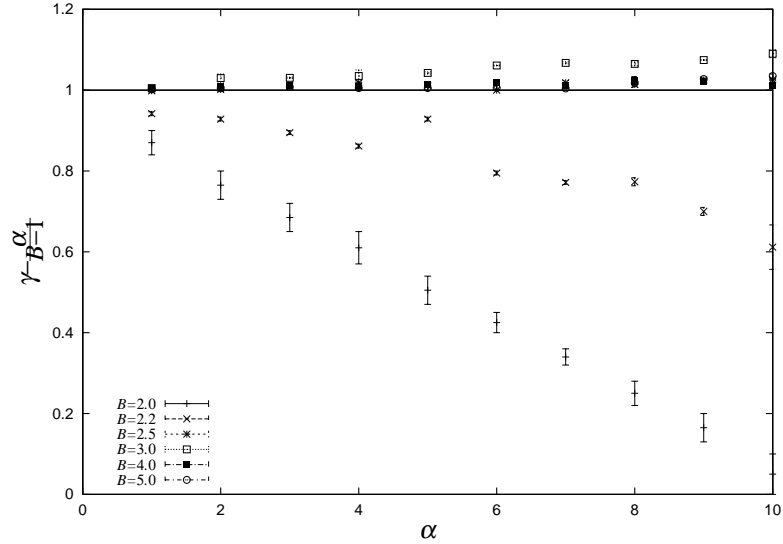

Fig. 8 Relationship between $\gamma$ and $\alpha$ and $B$.

process for the correlation function in the forthcoming paper.

From the viewpoint of physical observation, the distributional limit theorems, namely, the Mittag-Leffler distribution and the generalized arcsine law and the stable law, suggest that one can characterize the behaviour of non-stationary phenomena through the distribution of the time average. It is important to know what class the observation function belongs to, that is, whether the observation function is the $L_{l o c, m}^{1}(0,1)$ function with finite mean or not. Because the observation function in physical systems is not always the $L^{1}(m)$ function. Actually, we show that the correlation function is a typical example of the $L_{l o c, m}^{1}(0,1)$ function with finite mean. This means that the correlation function, or the statistical quantities based on the time average in the intermittent phenomena, is intrinsically random. Universal distributions for the time average of various observation functions are shown in Table 1

In the concept of the "ergodicity" proposed by L. Boltzmann, the ergodicity, i.e., the time average equals to the space average, guarantees the existence of the equilibrium state in dynamical systems. In the non-equilibrium steady state, Sinai-Ruelle-Bowen measure is considered to describe the non-equilibrium steady state. However, there are no concepts of the "ergodicity" in the non-equilibrium state, that is, the measure of the non-equilibrium nonstationary state is not at all elucidated on the basis of the time average of the dynamical systems. 8 We hope that randomness of the time average in infinite measure systems will give us the motive argument toward the ergodic problem in the non-equilibrium state. There is a possibility that Mittag-Leffler distribution or the generalized arcsine distribution or the stable distirbution could become one of the measures characterizing the non-equilibrium state. Actually, these distribution universally appear in diffusion and its generalizations.

8 From the aspect of the ensemble average, the approach to equilibrium of the observables is clearly shown using infinite measure systems in 21,22,26]. 
Table 1 Universal distributions of the time average of the observation function $g(x)$.

\begin{tabular}{lll}
\hline Invariant measure & $g(x)$ & Distribution \\
\hline Finite $(B<2)$ & $L^{1}(m)$ & Delta \\
Finite $(B<2)$ & $L_{l o c}^{1}(m)$ with infinite mean & Stable \\
Infinite $(B \geq 2)$ & $L^{1}(m)$ & Mittag-Leffler \\
Infinite $(B \geq 2)$ & $L_{l o c, m}^{1}$ with finite mean & Generalized arcsine \\
Infinite $(B \geq 2)$ & $L_{l o c, m}^{1}$ with infinite mean & Stable \\
\hline
\end{tabular}

Moreover, the generalized arcsine law has drawn much attention in disordered systems [27,28].

Acknowledgements T.A. would like to thank Y. Aizawa and T. Inoue for fruitful discussions. T.A. is supported by a grant to The 21st Century COE Program (Physics of SelfOrganization Systems) at Waseda University from the Ministry of Education, Culture, Sports, Science and Technology, Japan.

\section{A The distribution for the time average of the non- $L^{1}(m)$ function in the case of the finite measure}

In the case of the finite measure $(B<2)$, the invariant density can be written as

$\rho(x)=\frac{2-B}{2}\left\{x^{1-B}+(1-x)^{1-B}\right\}$.

Birkhoff's ergodic theorem 29 tells us that the p.d.f. of the sequence $\left\{T x, T^{2} x, \cdots, T^{n} x\right\}$ obeys the invariant density as $n \rightarrow \infty$. Let $\mathbf{X}_{n}$ be random variables with p.d.f. (40) and $g(x)=x^{-\alpha}(\alpha \geq 2-B)$. The distribution of $\mathbf{Y}_{n}=g\left(\mathbf{X}_{n}\right)$ is given by

$$
\begin{aligned}
F(x)=\operatorname{Pr}(\mathbf{Y}<x) & =\operatorname{Pr}\left(\mathbf{X}>x^{-\frac{1}{\alpha}}\right) \\
& =1-x^{-\frac{2-B}{\alpha}} .
\end{aligned}
$$

The general central limit theorem [30] says that the random variable $\left(\mathbf{Y}_{1}+\cdots+\mathbf{Y}_{n}\right) / n^{\alpha /(2-B)}$ has the stable distribution. Therefore

$\frac{1}{n^{\frac{\alpha}{2-B}}} \sum_{k=0}^{n-1} g\left(T^{k} \cdot\right) \rightarrow G_{(2-B) / \alpha}$ as $n \rightarrow \infty$,

where $G_{(2-B) / \alpha}$ is the stable distribution with index (2$B) / \alpha$.

\section{References}

1. P. Manneville, Intermittency, self similarity and $1 / f$ spectrum, Le Journal de Physique 41 1235-1243 (1980).

2. G. Ahlers and R. P. Behringer, The Rayleigh-Bénard instability and the evolution of turbulence, Prog. Theor. Phys. Suppl. 64, 186-201 (1978).

3. Y. Pomeau et al, Intermittent behavior in the BelousovZhabotinsky reaction, Le Journal de Physique Lettres 42 271-273 (1981).

4. M. Nirmal et al, Fluorescence intermittency in single cadmium selenid nanocrystals, Nature 383, 802-804 (1996).
5. F. Omori, On the aftershocks of earthquakes, J. College Sci., Imp. Univ. Tokyo, 7, 111-200 (1894).

6. M. Bottiglieri and C. Godano, On-off intermittency in eqrthquake occurrence, Phys. Rev. E 75, 026101 (2007).

7. Y. Aizawa, Y. Kikuchi, T. Harayama, K. Yamamoto, M. Ota and K. Tanaka, Stagnant motions in Hamiltonian Systems, Prog. Theor. Phys. Suppl. No. 98, 36-82 (1989).

8. Y. Aizawa, On the $f^{-1}$ spectral chaos, Prog. Theor. Phys. 72, 659-661 (1984)

9. X. Brokmann et al, Statistical aging and nonergodicity in the fluorescence of single nanocrystals, Phys. Rev. Lett. 90, 12061 (2003).

10. G. Margolin and E. Barkai, Nonergodicity of a time series obeying lévy statistics, J. Stat. Phys. 122 137-167 (2006).

11. D. A. Darling and M. Kac, On occupation times for Markov processes, Trans. Amer. Math. Soc. 84, 444-458 (1957).

12. J. Aaronson, An Introduction to Infinite Ergodic Theory (American Mathematical Society, 1997).

13. M. Thaler, The Dynkin-Lamperti arc-sine laws for measure preserving transformations, Trans. Amer. Math. Soc. 350, 4593-4607 (1998).

14. M. Thaler and R. Zweimüller, Distributional limit theorems in infinite ergodic theory, Probab. Theory Relat. Fields 135, 15-52 (2006).

15. M. Thaler, A limit theorem for sojourns near indifferent fixed points of one dimensional maps, Ergo. Th. ES Dynam. Sys., 22, 1289-1312 (2002).

16. G. Bel and E. Barkai, Weak ergodicity breaking with deterministic dynamics, Europhys. Lett., 74, 15-21 (2006).

17. Y. Aizawa, Comments on the non-stationary chaos, Chaos, Soliton \& Fractals 11, 263-268 (2000).

18. M. Thaler, Transformations on $[0,1]$ with infinite invariant measures, Isr. J. Math. 46, 67-96 (1983).

19. D. R. Cox, Renewal Theory (Methuen, New York, 1962).

20. Y. Aizawa and T. Kohyama, Kyoto Summer Institute "Chaos and Statistical Mechanics", ed. Y. Kuramoto, 109116, Springer, (1983).

21. T. Akimoto and Y. Aizawa, Scaling exponents of the slow relaxation in non-hyperbolic chaotic dynamics, NPCS 6 , 178-182 (2006).

22. T. Akimoto and Y. Aizawa, New aspects of the correlation functions in non-hyperbolic chatic systems, J. K. Phys. Soc. 50, 254-260 (2007).

23. Y. Aizawa, Non-stationary chaos revisited from large deviation theory, Prog. Theor. Phys. Suppl. No. 99, 149-164 (1989).

24. J. Lamperti, An occupation time theorem for a class of stochastic proceses, Trans. Amer. Math. Soc. 88, 380-387 (1958).

25. M. Thaler, The asymptotics of the Perron-Frobenius operator of a class of interval maps preserving infinite measures, Studia Math. 143, 103-119 (2000).

26. S. Tasaki and P. Gaspard, Spectral properties of a piecewise linear intermittent map, J. Stat. Phys. 109, 803-820 (2002).

27. E. Barkai, Residence time statistics for normal and fractional diffusion in a force field, J. Stat. Phys. 123, 883-907 (2006). 
28. S. Burnov and E. Barkai, Occupation time statistics in the quenched trap model, Phys. Rev. Let. 98, 250601 (2007).

29. G. D. Birkhoff, Proof of the ergodic theorem, Proc. Nat. Acad. Sci. USA, 17, 656-660 (1931).

30. W. Feller, An Introduction to Probability Theory and Its Applications, second ed., Vol. II, 448. Wiley, (1971). 